punkt beruhte („Stoßzahlansatz“). Dies entspricht genau dem in Abschnitt II durchgeführten Verfahren bei der Konstruktion repräsentativer Ensemble, wobei die Verteilungsfunktion $f$ als selbstkonsistente Größe angesehen wird. Das Boltzmannsche $H$-Theorem erweist sich so als echter Spezialfall des in $\mathrm{Ab}$ schnitt II gefundenen Anwachsens der Entropie $S_{A}$ einer reduzierten, selbstkonsistenten Größe $A$.
Für Fälle mit allgemeineren Wechselwirkungen, wie z. B. Plasmen, wo langreichweitige CoulombKräfte vorhanden sind, liegen die Verhältnisse jedoch wesentlich komplizierter und eine schlüssige Antwort auf die eingangs gestellten Fragen existiert bis heute nicht.

Herrn Prof. J. Yvon und Herrn Dr. D. Pfirsch danken wir für interessante Diskussionen.

\title{
Die gasionisierende Strahlung einer Entladung in $\mathrm{N}_{2}-\mathrm{O}_{2}$-Gemischen
}

\author{
(Quantitativer Nachweis kleiner $\mathrm{O}_{2}$-Konzentrationen in $\mathrm{N}_{2}$ ) \\ Von A. Przybylski \\ Aus dem Institut für Angewandte Physik der Universität Hamburg \\ (Z. Naturforschg. 16 a, 1232-1237 [1961]; eingegangen am 14. August 1961)
}

\begin{abstract}
This paper gives a method to trace quantitatively small quantities of oxygen (down to $10^{-5}$ parts ir. 1) in mixtures of oxygen and nitrogen. This is based on the effect of the ionization of oxygen molecules in a mixture of oxygen and nitrogen by radiation emitted by nitrogen molecules with ionization potentials $U_{\mathrm{O}_{2}}<U_{\mathrm{N}_{2}}$ and the energy of radiation between $U_{\mathrm{O}_{2}}$ and $U_{\mathrm{N}_{2}}$.

Coefficients of absorption and intensities of this ionizing radiation are given in dependence on the concentration of oxygen.

A radiation emitted by nitrogen and ionizing nitrogen itself is traced and its coefficient of absorption $\left(\mu \approx 750 \mathrm{~cm}^{-1}\right)$ and its intensity are measured.
\end{abstract}

Unterhält man in einem Gas eine elektrische Entladung, dann werden durch unelastische Elektronenstöße die Moleküle dieses Gases teils ionisiert und teils zur Emission von Photonen angeregt. Besteht dieses Gas aus zwei Sorten von Molekülen, deren Ionisierungsenergien verschieden groß sind, z. B. dem hier untersuchten Gemisch von $\mathrm{O}_{2}$ und $\mathrm{N}_{2}\left(U_{\mathrm{O}_{2}}\right.$ $=12,075 \mathrm{eV}$ nach $^{1} ; U_{\mathrm{N}_{2}}=15,58 \mathrm{eV}$ nach $^{2}$ ), dann kann eine von den Stickstoffmolekülen emittierte Strahlung, deren Energie zwischen den beiden Ionisierungsenergien liegt, die Sauerstoffmoleküle ionisieren. Es ergibt sich, daß dieser Effekt es ermöglicht, quantitative Messungen kleiner Sauerstoffkonzentrationen in Stickstoff durchzuführen ${ }^{3}$.

\section{Apparatur und Meßmethode}

Die Abb. 1 zeigt die Versuchsanordnung, bestehend aus einer Entladungsstrecke und einer Ionisationskammer zum Nachweis der durch die ionisierende Strahlung gebildeten Ladungsträger. Die Entladungsstrecke besteht aus einer koaxialen Zylinderanordnung. Der äußere Zylindermantel ist die Kathode, der Draht in der Achse die Anode. Durch ein seitliches Gitterfenster

\footnotetext{
1 K. Watanabe, J. Chem. Phys. 26, 542 [1957].

2 H. Herzberg, Molecular Spectra, D. van Nostrand Comp., New York 1950.
}

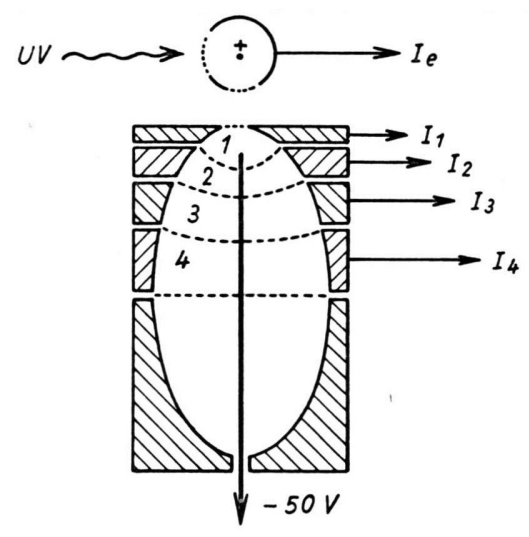

Abb. 1. Versuchsanordnung (schematisch) bestehend aus der Entladungsstrecke (koaxiales Zylinderfeld) und einer unterteilten Ionisationskammer mit der Kathode als Innenelektrode.

im Mantelrohr wird UV-Licht eines Quecksilberbrenners eingestrahlt, das Photoelektronen für die Unterhaltung der Entladung auslöst. Durch ein Gitterfenster am Boden des Außenzylinders kann die Entladungsstrahlung in die Ionisationskammer treten.

Die Anode der Ionisationskammer hat die Form eines Rotationsellipsoids, in dessen Achse sich ein dünner Draht als Kathode befindet. Diese geometrische An-

\footnotetext{
${ }^{3}$ Die Zusammenfassung eines Teils der Untersuchungen von $\mathrm{N}_{2}-\mathrm{O}_{2}$ Gemischen ist in Proc. 4. Int. Conf. on Ionization Phenomena in Gases, North Holland Publ. Comp., Amsterdam 1960, S. IC 215 erschienen.
} 
ordnung ergibt einen besonders übersichtlichen Feldverlauf in der Kammer. Die kleine Oberfläche der Kathode reduziert einen Oberflächenphotoeffekt, durch den auch langwellige Photonen, die nicht ionisieren können, zum Kammerstrom beitragen könnten. Die Kammer ist in vier Segmente aufgeteilt. Dadurch ist es möglich, die in den Raumgebieten 1 bis 4 gebildeten Ionen getrennt zu messen. Aus den Quotienten $I_{m} / I_{n}$ ( $I_{j}$ ist der Strom im $j$-ten Segment) lassen sich die Absorptionskoeffizienten $k_{m n}$ bei dem Meßdruck $p$ bestimmen.

Wird in der Kammer eine monochromatische Strahlung absorbiert, deren Absorptionskoeffizient, bezogen auf 760 Torr, gleich $\mu \mathrm{cm}^{-1}$ ist, dann wird

$$
k_{23}=k_{34}=\mu \cdot p / 760 \quad(\text { BeERsches Gesetz }) \text {. }
$$

Wird dagegen ein Strahlengemisch absorbiert, dessen Komponenten verschieden große $\mu$ haben, so wird sich wegen der Ausfilterung der weicheren Komponenten $k_{23}>k_{34}$ ergeben.

Ist nun $k=\mu \cdot p / 760$ der Absorptionskoeffizient beim Druck $p, x_{2}$ die Entfernung vom Anodendraht der Entladungsstrecke zum Anfang des zweiten Segments, $x_{5}$ die Entfernung bis zum Ende des vierten Segments, $L_{0}$ die Zahl der von der Entladung in den Raumwinkel $\Omega$, unter dem das Eintrittsfenster der Ionisationskammer von der Strahlungsquelle aus erscheint, emittierten ionisierenden Photonen, dann ist die Zahl $L$ der in den Segmenten 2, 3 und 4 absorbierten Photonen gleich

$$
L=L_{0}\left(e^{-k x_{2}}-e^{-k x_{5}}\right) .
$$

Der Quotient $L / L_{0}$ ist in Abhängigkeit von $k$ für die vorliegende geometrische Anordnung $\left(x_{2}=2,85\right.$ $\left.\mathrm{cm} ; x_{5}=5,2 \mathrm{~cm}\right)$ in Abb. 2 aufgetragen. Der Verlauf dieser Kurve ist so zu verstehen, daß bei großen $k$ nur wenig Strahlung bis zur Ionisationskammer vordringt, bei kleinen $k$ die Strahlung die Kammer fast ungeschwächt erreicht und diese nahezu ungeschwächt wieder verläßt. Dazwischen liegt ein Maximum der Zahl der absorbierten Photonen. Da der Kammerstrom der Zahl der absorbierten Photonen und die Zahl der emittierten Photonen in erster Näherung dem Entladungsstrom $I_{\mathrm{e}}$ proportional ist, ergibt sich somit

$$
L / L_{0}=A \cdot\left(\Sigma I_{i}\right) / I_{\mathrm{e}},
$$

$\sum I_{i}=I_{2}+I_{3}+I_{4}$ Gesamtstrom in den Segmenten 2, 3 und $4 ; I_{\mathrm{e}}$ Entladungsstrom, $A$ Proportionalitätsfaktor, der die Ausblendung und die Zahl der pro ionisierendem Stoß eines Elektrons emittierten ionisierenden Photonen berücksichtigt.

\footnotetext{
4 A. Przybylski, Z. Phys. 161, 264 [1958].
}

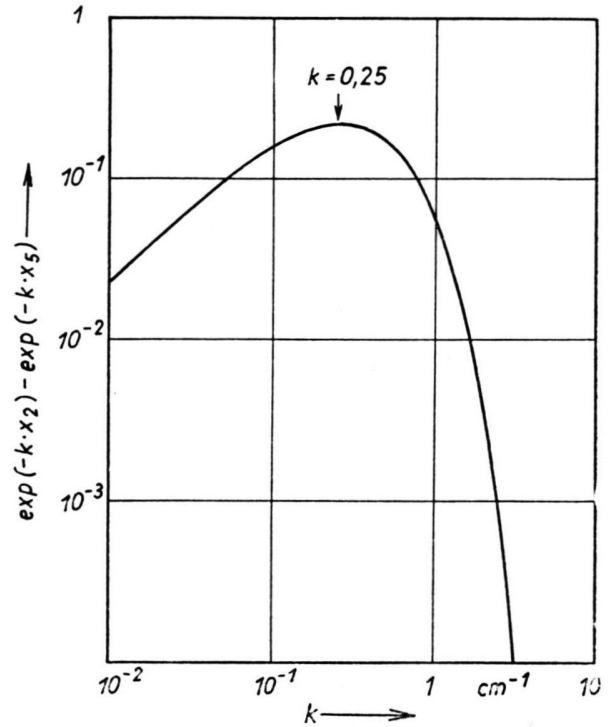

Abb. 2. In der Ionisationskammer absorbierter Anteil der Strahlung, die von der Entladung in die Kammer emittiert wird, in Abhängigkeit vom Absorptionskoeffizienten.

Da nun der Absorptionskoeffizient $k$ nach dem BeERschen Gesetz proportional zum Druck ist, wird für eine monochromatische Strahlung mit einheitlichem $\mu$ die Auftragung von $\left(\sum I_{i}\right) / I_{\mathrm{e}}$ gegen den Druck $p$ ebenfalls einen Verlauf wie Abb. 2 zeigen.

Eine detaillierte Darstellung der Meßmethode findet sich in ${ }^{4}$.

\section{Ergebnisse in einem Gemisch aus $\mathrm{N}_{2}$ und $\mathrm{O}_{2}$}

Die Abb. 3 zeigt Absorptionskoeffizienten, wie sie in Gemischen verschiedener Konzentrationen gemessen wurden. Die Kurve für Sauerstoff allein zeigt, daß bei Drucken $p>12$ Torr (gemessen bis 30 Torr) eine einzige monochromatische Strahlungskompo-

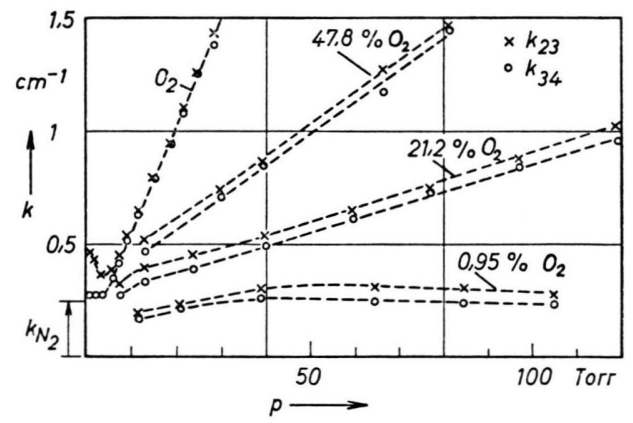

Abb. 3. Absorptionskoeffizienten von Gemischen aus Stickstoff und Sauerstoff. (Die angegebenen Zahlen geben den Volumenanteil von $\mathrm{O}_{2}$ an.) 
nente in die Ionisationskammer gelangt mit einem auf Atmosphärendruck reduzierten Absorptionskoeffizienten von $\mu=38 \mathrm{~cm}^{-1}$. Die Monochromasie folgt aus dem Zusammenfallen von $k_{23}$ und $k_{34}$, sowie aus der Erfüllung des Beerschen Gesetzes. Bei Drucken $p<10$ Torr gelangen weitere Strahlungskomponenten mit höheren $\mu$ in die Ionisationskammer, was aus der Aufspaltung von $k_{23}$ und $k_{34}$ folgt. Diese Absorptionskoeffizienten stellen die Absorption der $\mathrm{O}_{2}$-Strahlung in $\mathrm{O}_{2}$ dar.

Die Gemische ergeben bei gleichem Gesamtdruck durchweg kleinere Absorptionskoeffizienten. Die Absorptionskoeffizienten für Mischungen mit einem Gehalt von weniger als $1 \%$ Sauerstoff fallen mit den Werten der Kurve für $0,95 \% \mathrm{O}_{2}$ zusammen ${ }^{5}$. Dies weist darauf hin, daß bei diesen geringen Konzentrationen der Sauerstoff nicht mehr wesentlich an der Absorption beteiligt ist und der Stickstoff selbst die Absorption bestimmt. (Ob diese Absorption mit einer Ionisierung verbunden ist, wird weiter unten diskutiert.) Über eine eingehende Untersuchung des Sauerstoffs siehe ${ }^{4}$.

Die Kurven der Abb. 3 sagen aus, daß gegenüber den Verhältnissen in Sauerstoff allein in $\mathrm{O}_{2}-\mathrm{N}_{2}$ Gemischen Strahlungskomponenten wirksam sind, die vom Stickstoff emittiert werden. Dies läßt sich folgendermaßen zeigen:

In reinem Sauerstoff wird ein $k=1,5$ bei einem Druck von 30 Torr gemessen $(\mu=38)$. Würde nun in Gemischen (z. B. $\mathrm{O}_{2}: \mathrm{N}_{2}=1: 1$ ) der Stickstoff weder ionisierende Strahlung emittieren noch vom Sauerstoff emittierte Strahlung absorbieren, dann würde ein $\mathrm{Ab}$ sorptionskoeffizient $k=1,5$ stets bei dem gleichen Sauerstoff-Partialdruck $P_{\mathrm{O}_{2}}=30$ Torr gefunden werden. (Bei einer Mischung im Verhältnis 1:1 also bei einem Gesamtdruck von $p_{\mathrm{g}}=60$ Torr.) Bewirkt nun aber der Stickstoffanteil eine zusätzliche Absorption von Sauerstoffstrahlung, dann wird $k=1,5$ schon bei einem niedrigeren Sauerstoff-Partialdruck als $p_{0_{2}}=30$ Torr erreicht (d. h. bei einem 1 : 1-Gemisch bei einem Gesamtdruck, der kleiner als 60 Torr ist). Tatsächlich mißt man in 1 : 1-Gemischen jedoch erst bei einem $\mathrm{O}_{2}$-Partialdruck von 42,5 Torr $\left(p_{\mathrm{g}}=85\right.$ Torr) einen Absorptionskoeffizienten $k=1,5$. Es muß hier also eine neue, von der Sauerstoffstrahlung verschiedene Strahlungskomponente hinzutreten, die offensichtlich vom Stickstoff emittiert wird und den vergleichsweise niedrigeren Absorptionskoeffizienten bewirkt. Die Verletzung des BeErschen Gesetzes (vgl. Abb. 3) weist ebenfalls darauf hin, daß in $\mathrm{N}_{2}-\mathrm{O}_{2}$-Gemischen nicht lediglich monochromatische Sauerstoffstrahlung absorbiert wird.

Die Zusammensetzung von $k$ in einem $\mathrm{O}_{2}-\mathrm{N}_{2}$ Gemisch und bei Gesamtdrucken über 10 Torr in Abhängigkeit von der Sauerstoffkonzentration kann folgendermaßen dargestellt werden:

$$
k=k_{\mathrm{N}_{2}}+\mu_{\mathrm{O}_{2}} p_{\mathrm{O}_{2}} / 760
$$

mit $k_{\mathrm{N}_{2}}=0,3 \mathrm{~cm}^{-1} ; \mu_{02}=25 \mathrm{~cm}^{-1}$ (gegen Stickstoffstrahlung; bezogen auf 760 Torr $\left.\mathrm{O}_{2}\right) ; p_{02}=$ Sauerstoffpartialdruck in Torr.

Der vom Stickstoff herrührende Anteil ist in dem aufgetragenen Druckbereich praktisch unabhängig vom Druck; er sei mit $k_{\mathrm{N}_{2}}$ bezeichnet.

Diese Druckabhängigkeit würde sich durch eine überlagerte Absorption mehrerer Strahlungskomponenten mit wenig verschiedenen $\mu$ erklären lassen; durch entsprechende Ausfilterung könnte ein scheinbar vom Druck nicht abhängendes $k_{N_{2}}$ bewirkt werden. Die $k$-Kurve für die Gemische mit 47,8\% $\mathrm{O}_{2}$ und $21,2 \% \mathrm{O}_{2}$ sind $\mathrm{Ge}$ raden (vgl. Abb. 3), deren Verlängerungen gegen $p=0$ etwa bei dem oben genannten $k_{\mathrm{N}_{2}}$-Wert münden.

Wie oben bereits gesagt, muß man zur Deutung der scheinbaren Druckunabhängigkeit von $k_{\mathrm{N}_{2}}$ (im Druckbereich 12-100 Torr) die Absorption mehrerer Komponenten einer Stickstoffbestrahlung durch Stickstoff annehmen. Diese Komponenten zeigen in Stickstoff verschiedene $\mu$ und bewirken so wegen der verschieden starken Ausfilterung ein fast konstantes $k_{\mathrm{N}_{2}}$. (Wegen der Ausfilterung hängt die Größe von $k_{\mathrm{N}_{2}}$ von der Entfernung der Strahlungsquelle zur Meßstelle ab.) Wie die Erfüllung des BeErschen Gesetzes für den Sauerstoffanteil des Absorptionskoeffizienten der Stickstoffstrahlung zeigt, haben die verschiedenen Strahlungskomponenten gegenüber $\mathrm{O}_{2}$ ein einheitliches $\mu_{0_{2}}=25$ $\mathrm{cm}^{-1}$. Dies widerspricht nicht dem Tatbestand, daß diese Komponenten der Stickstoffstrahlung durch den Stickstoff verschieden stark absorbiert werden, da Resonanzlinien des Stickstoffs wohl vom Stickstoff verschieden stark, im Absorptionskontinuum des Sauerstoffs aber alle gleich stark absorbiert werden können.

Zusätzlich läßt sich aus den $k$-Kurven für reinen Sauerstoff und für das Gemisch mit 47,8\% $\mathrm{O}_{2}$ entnehmen, daß in diesem Gemisch die Sauerstoffstrahlung mit $\mu=38 \mathrm{~cm}^{-1}$ nicht wesentlich vorhanden ist, da der in dieser Kurve enthaltene Sauerstoffanteil an der Absorption gemäß (4) völlig mit $\mu_{\mathrm{O}_{2}}=25$ beschrieben wird. Eine zusätzliche $\mathrm{Ab}$ sorption der Sauerstoffstrahlung mit $\mu=38 \mathrm{~cm}^{-1}$ würde dieses Verhalten stören, falls die Sauerstoffstrahlung mit einer der Stickstoffstrahlung vergleichbaren Intensität auftreten würde.

Es entsteht jetzt noch die Frage, ob die vom Stickstoff emittierte Strahlung sowohl $\mathrm{O}_{2}$ als auch $\mathrm{N}_{2}$ oder nur $\mathrm{O}_{2}$ ionisiert. Um diese Frage zu klären, wurde der Quotient $\left(\Sigma I_{i}\right) / I_{\mathrm{e}}$ aus dem Gesamtstrom in der Ionisationskammer und dem Entladungsstrom in Abhängigkeit von der Sauerstoffkonzentration ge-

\footnotetext{
5 Die Prozentangaben sind Vol.-Prozent.
} 


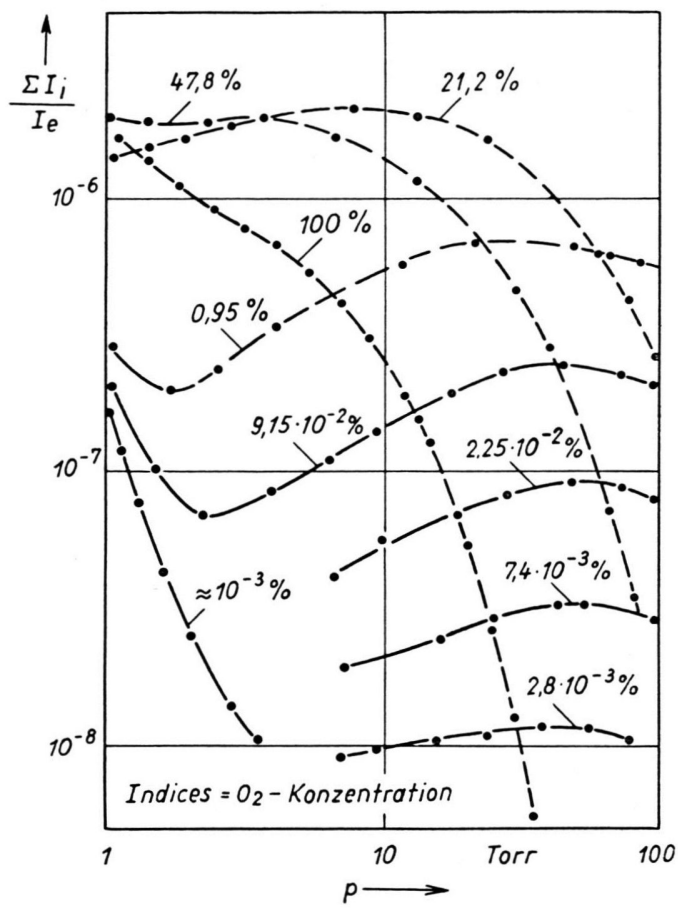

Abb. 4. $\left(\Sigma I_{i}\right) / I_{\mathrm{e}}$ für Gemische aus Stickstoff und Sauerstoff. Kurvenparameter ist die $\mathrm{O}_{2}$-Konzentration.

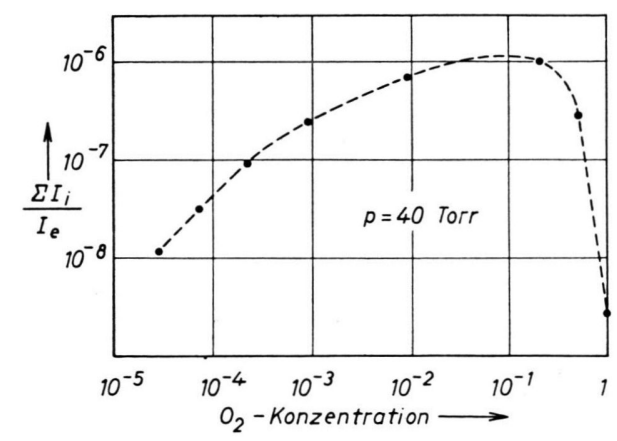

Abb. 5. Auszug aus Abb. 4. $\left(\sum I_{i}\right) / I_{\mathrm{e}}$ für einen Gesamtdruck von 40 Torr und konstantes $E / p$ in Abhängigkeit von der Sauerstoffkonzentration.

messen. Wie Abb. 4 zeigt, hängt $\left(\sum I_{i}\right) / I_{\mathrm{e}}$ bei kleinen Sauerstoffkonzentrationen sehr stark von diesen ab. In Abb. 5 ist nun $\left(\Sigma I_{i}\right) / I_{\mathrm{e}}$ für einen Gesamtdruck von 40 Torr in Abhängigkeit von der Sauerstoffkonzentration aufgetragen. Daraus ersieht man, daß für kleine Sauerstoffkonzentrationen ein linearer Zusammenhang zwischen diesen und $\left(\sum I_{i}\right) / I_{\mathrm{e}}$ besteht. Wie eine nähere Diskussion (siehe Anhang)

\footnotetext{
6 Vorläufige Messungen in einem Gemisch aus $\mathrm{H}_{2}$ und $\mathrm{O}_{2}$ ergeben ein ähnliches Bild wie die Ergebnisse in $\mathrm{N}_{2}$ und $\mathrm{O}_{2}$.
}

zeigt, bedeutet dieser Zusammenhang, daß die Stickstoffstrahlung nur den Sauerstoff ionisiert ${ }^{6}$.

\section{Anwendung der Photoionisierung zur Bestimmung von $\mathrm{O}_{2}$ in $\mathrm{N}_{2}$}

Um mit Hilfe des beschriebenen Effektes kleine Sauerstoffkonzentrationen in Stickstoff quantitativ zu bestimmen, ist folgendes zu beachten :

Die beschriebene Meßanordnung ist zunächst mit Gasgemischen bekannter Konzentrationen zu eichen. Mit Hilfe einer Eichkurve wie Abb. 5 läßt sich dann der Sauerstoffgehalt unbekannter $\mathrm{N}_{2}-\mathrm{O}_{2}$-Gemische bestimmen. Weiterhin sind die Meßwerte bei einheitlicher Spannung an der Entladungsstrecke, also bei etwa gleichem Entladungsstrom und bei jeweils gleichem Gesamtdruck aufzunehmen. Diese Maßnahme ist notwendig, um eine eindeutige Beziehung zwischen $\left(\Sigma I_{i}\right) / I_{\mathrm{e}}$ und der Sauerstoffkonzentration zu erhalten, da sich bei konstantem Druck und veränderter Spannung $U$, also einer Variation von $E / p$ am Draht des Entladungsrohres, der Quotient $\left(\sum I_{i}\right) / I_{\mathrm{e}}$ ändert. Eine Untersuchung über diese Änderung findet sich in ${ }^{7}$. Bei konstanten Bedingungen ist es für diese Messungen unerheblich, ob die Entladung unselbständig oder selbständig (Korona) ist.

Der Druck von 40 Torr wurde hier gewählt, da die Kurven der Abb. 4 etwa bei diesem Druck ein Maximum besitzen, der Wert $\left(\Sigma I_{i}\right) / I_{\mathrm{e}}$ also gegen geringe Fehler in der Druckmessung unempfindlich ist.

Der vorerwähnte lineare Zusammenhang zwischen dem Sauerstoffgehalt und $\left(\Sigma I_{i}\right) / I_{\mathrm{e}}$ ist im Falle des $\mathrm{N}_{2}-\mathrm{O}_{2}$-Gemisches bis zu sehr kleinen Sauerstoffkonzentrationen gegeben, da in dem betreffenden Druckbereich keine Stickstoffstrahlung in die Kammer gelangt, die den Stickstoff selbst ionisiert. Bei der Anwendung der vorliegenden Methode auf andere Gasgemische ist demnach die Wahl des für die Messungen zu wählenden Gesamtdruckes unter dem Gesichtspunkt zu treffen, daß dort keine das Hauptgas selbst ionisierende Strahlung in die Ionisationskammer gelangt.

Eine Störung des beschriebenen Meßverfahrens kann durch Gasbeimischungen erfolgen, die eine kleinere Ionisierungsenergie als der Stickstoff haben

7 A. Przybylski, Z. Phys., in Vorbereitung. 
und einen hinreichend großen Absorptionskoeffizienten gegenüber der Stickstoffstrahlung besitzen.

Der Vorteil des Nachweisverfahrens liegt in einer sich schnell einstellenden, kontinuierlichen und quantitativen Anzeige des Sauerstoffgehalts. Wegen anderer Verfahren zum Nachweis von $\mathrm{O}_{2}$ in $\mathrm{N}_{2}$ siehe z. B. ${ }^{8}$.

\section{Ergebnisse in Stickstoff}

Den Meßwerten für $\mathrm{N}_{2}-\mathrm{O}_{2}$-Gemische mit sehr kleinen Sauerstoffkonzentrationen läßt sich das Verhalten der gasionisierenden Strahlung in reinem Stickstoff entnehmen. Wie in Abb. 4 zu ersehen, streben die $\left(\Sigma I_{i}\right) / I_{\mathrm{e}}$-Kurven für Sauerstoffkonzentrationen unterhalb $1 \%$ bei Gesamtdrucken unter 5 Torr asymptotisch gegen eine Grenzkurve, werden also unabhängig vom Sauerstoffzusatz. Das bedeutet also, daß hier eine vom Stickstoff emittierte Strahlung wirksam wird, die den Stickstoff selbst ionisiert. Der sauberste zur Verfügung stehende, "nachgereinigte“ Stickstoff hatte eine Sauerstoffverunreinigung von ca. $10^{-5}$. Bei kleinen Drucken unterhalb 2 Torr sind die $\left(\sum I_{i}\right) / I_{\mathrm{e}}$-Werte praktisch die Grenzkurve. Für höhere Drucke jedoch ist die Sauerstoffverunreinigung maßgebend für die Meßwerte.

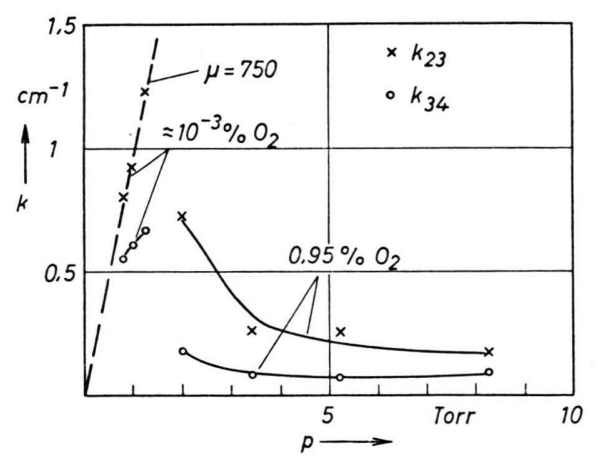

Abb. 6. Absorptionskoeffizienten von Stickstoff-SauerstoffGemischen bei kleinen Drucken und kleinen Sauerstoffkonzentrationen.

Der Absorptionskoeffizient dieser eigenionisierenden Stickstoffstrahlung ergibt sich aus Abb. 6. Die bei einer Sauerstoffkonzentration von ca. $1 \%$ bis zu einem Druck von etwa 4 Torr flach verlaufenden $k$-Werte wachsen bei kleineren Drucken beträchtlich an, entsprechend dem Ansteigen von $\left(\Sigma I_{i}\right) / I_{\mathrm{e}}$ in

8 A. Klemenc, Die Behandlung und Reindarstellung von Gasen, Springer-Verlag, Wien 1948.

9 G. R. Bainbridge u. W. A. Prowse, Canad. J. Phys. 34. 1038 [1956].
Abb. 4. Für eine $\mathrm{O}_{2}$-Konzentration von etwa $10^{-5}$, bei der sich $k$ aus Intensitätsgründen nur bei ganz kleinen Drucken messen läßt, ist diese Aufsteilung sehr deutlich. Der Absorptionskoeffizient dieser Strahlung kann abgeschätzt werden. Wie Abb. 6 zeigt, dürfte

$$
\mu=750 \mathrm{~cm}^{-1}
$$

etwa die Grenzkurve sein. Diese Strahlung hat ein $\delta / \alpha$ von etwa

$$
\delta / \alpha \approx 10^{-3},
$$

wobei $\delta$ die Zahl der pro $\mathrm{cm}$ durch Elektronenstoß emittierten Photonen ist.

In der Literatur findet sich ein Wert für den $\mathrm{Ab}$ sorptionskoeffizienten von eigenionisierender Stickstoffstrahlung. Bainbridge und Prowse ${ }^{9}$ geben ein $\mu=0,25$ $\mathrm{cm}^{-1}$ an, das sie mit einer Funkenentladung bei Drucken von $460<p<760$ Torr gemessen haben. Bei diesen Drucken wäre eine Strahlung mit $\mu=750 \mathrm{~cm}^{-1}$ wegen der großen Absorption nicht nachzuweisen gewesen. Der von diesen Autoren gefundene Wert von $\mu=0,25 \mathrm{~cm}^{-1}$ läßt die Vermutung $\mathrm{zu}, \mathrm{da} ß$ es sich hier um einen Wert handelt, der dem $k_{\mathrm{N}_{2}}$ in $\mathrm{O}_{2}-\mathrm{N}_{2}$-Gemischen entspricht, wie er in dieser Arbeit gemessen wurde, und der der Ionisierung von $\mathrm{O}_{2}$ durch $\mathrm{N}_{2}$-Strahlung zuzuordnen ist. Da $k_{\mathrm{N}_{2}}$ bei kleinen Sauerstoffverunreinigungen des Stickstoffs von diesen fast unabhängig ist, kann wegen der dann auftretenden Reproduzierbarkeit leicht der Eindruck entstehen, es handle sich um einen reinen Stickstoffeffekt. Ein Vergleich mit den bei $W_{\text {EISSLER }}{ }^{10}$ angegebenen $\mu$ zeigt, da $\beta$ ein kleinerer Wert für $\mu$ bei Stickstoff auch kaum zu erwarten ist, da die $\mu$ für kontinuierliche Absorption schon sehr nahe an der Ionisierungsgrenze ein Maximum $\mu \approx 650$ $\mathrm{cm}^{-1}$ bei etwa $750 \AA$ A erreichen. Die $\mu$ für Resonanzlinien dürften also über $650 \mathrm{~cm}^{-1}$ liegen.

\section{Anhang}

Der lineare Zusammenhang von $\left(\Sigma I_{i}\right) / I_{\mathrm{e}}$ und dem Sauerstoffpartialdruck in Gemischen mit kleinen $\mathrm{O}_{2}$ Konzentrationen, wie ihn die Abb. 5 zeigt, läßt sich durch eine Fallunterscheidung klären.

In dem $\mathrm{N}_{2}-\mathrm{O}_{2}$-Gemisch können folgende Photoionisierungsprozesse auftreten:

a) Stickstoffstrahlung ionisiert den Sauerstoff;

b) Sauerstoffstrahlung wird sowohl von Stickstoff als auch von Sauerstoff absorbiert, ionisiert aber nur den Sauerstoff ;

c) Sauerstoffstrahlung ionisiert sowohl Sauerstoff als auch Stickstoff;

${ }^{10}$ G. L. WeIssLer, Handbuch der Physik. Bd. XXI, SpringerVerlag 1956. 